\title{
Rituximab-Induced Serum Sickness in a Patient with Pemphigus Vulgaris: A Case Report
}

\author{
Mohamad Khatib ${ }^{1}$, Solaiman Allafi ${ }^{1}$, and Abdulqadir Nashwan ${ }^{1}$ \\ ${ }^{1}$ Hamad Medical Corporation
}

September 10, 2020

\begin{abstract}
Serum sickness, a reaction characterized by fever, rash, myalgia, and arthralgia, can occur in patients who receive chimeric monoclonal antibody therapy. Serum sickness is considered as a rare adverse event following rituximab therapy. We report a case of serum sickness in a newly treated patient with rituximab for pemphigus vulgaris.
\end{abstract}

\section{Key Clinical Message}

Clinicians should be vigilant for potential acute or delayed serum sickness as possible adverse reactions to weigh the risks and benefits of continuing/reintroducing rituximab for patients with pemphigus vulgaris.

\section{Background}

Monoclonal antibodies have been used for the modulation of immune responses in several disorders, including cancer, autoimmune, and infectious diseases. ${ }^{1}$

Rituximab (RTX) is a chimeric monoclonal antibody that specifically binds to the B-cell surface antigen CD20 which produces antibody-dependent cell- and complement-mediated cytotoxicity in these cells. ${ }^{2-4}$ The mechanism of action of RTX including, regulating cell-cycle signaling, inducing apoptosis, improving cells' sensitization to cytotoxic drugs, complement-mediated cytotoxicity, and antibody-dependent cellular cytotoxicity ${ }^{4}$

RTX has been used in the treatment of previously untreated or previously treated CD20-positive chronic lymphocytic leukemia (CLL) in adults ${ }^{5}$, granulomatosis with polyangiitis ${ }^{6}$, microscopic polyangiitis ${ }^{7}$, nonHodgkin lymphomas ${ }^{8}$, pemphigus vulgaris ${ }^{9}$, rheumatoid arthritis ${ }^{10}$, and other off-label uses such as acquired thrombotic thrombocytopenic purpura ${ }^{11}$, Waldenström macroglobulinemia ${ }^{12}$, refractory autoimmune hemolytic anemia ${ }^{13}$, etc.

As a single agent, RTX (1000 mg) is usually administered weekly for 4 weeks. When used in combination with chemotherapy, it is often administered every 3 to 4 weeks. The pharmacokinetics of RTX is like that seen with human immunoglobulin $\mathrm{G}$ (IgG). ${ }^{14}$

Pemphigus is an autoimmune disease characterized by blisters and erosions of the skin and mucosal membranes. It is mediated by autoantibodies that target the transmembranous adhesion glycoproteins desmoglein 1 (Dsg1) and desmoglein 3 (Dsg3). Pemphigus Vulgaris (PV) is the major subtype of Pemphigus and is characterized as having a progressive course that leads to death if the disease is not treated..$^{15}$

Several previous studies have reported adverse events such as cough, dyspnea, fever, and erythema, as well as late adverse events such as severe infection and agranulocytosis in patients treated with RTX. ${ }^{16}$ 
Serum sickness is a rare hypersensitivity reaction due to foreign antigens, causing fever, rash, and arthralgia. ${ }^{17}$ RTX may produce human anti-chimeric antibodies (HACAs) and induce serum sickness. ${ }^{18}$ Although serum sickness is relatively common among autoimmune disease patients, there are few reports in patients with PV.

\section{Case Presentation}

A 33-year-old male was admitted with a history of fever, malaise, arthralgia, and myalgia for one day. Two weeks ago, he was started on RTX (1000mg IV 2 times per week) for the treatment of severe pemphigus vulgaris in addition to his previous treatment with intravenous immunoglobulin (IVIG) (2 g/ kg per cycle), azathioprine $(2.5 \mathrm{mg} / \mathrm{kg})$, and prednisolone $(2 \mathrm{mg} / \mathrm{kg}$ per day) which were started earlier. The symptoms appeared 11 days after the second dose.

Subsequent physical examination revealed fever (a temperature of 38.5degC), hemodynamic instability with sinus tachycardia of 130 - $140 \mathrm{bpm}$, mild tachypnea, malaise, generalized body rash (cutaneous blisters), and generalized body pain. He reported severe pain while moving his elbows, shoulders, knees, wrists, ankles, spine, metacarpophalangeal joints, and temporomandibular joints. However, no effusions, swelling, and erythema were noted. He was immediately started on a wide-spectrum antibiotic at the beginning in view of suspected sepsis and was discontinued shortly after admission.

Subsequent laboratory investigations revealed leukocyte count $26.4\left(4-10 \times 10^{\wedge} 3 / \mathrm{uL}\right)$, neutrophils $84.3 \%$, platelet count $267\left(150-400 \times 10^{\wedge} 3 / \mathrm{uL}\right)$, erythrocyte count $6.4\left(4.5-5.5 \times 10^{\wedge} 6 / \mathrm{uL}\right)$, C-reactive protein level $112(0.0-5.0 \mathrm{mg} / \mathrm{L})$, uric acid level $7.7(3.4-7.0 \mathrm{mg} / \mathrm{dL})$, creatinine $144(62-106 \mathrm{umol} / \mathrm{L})$. Coagulation profile, electrolytes, C3 and $\mathrm{C} 4$ levels within normal ranges, Urinalysis was within normal range except a trace of blood, and blood cultures and viral panel were negative.

Based on his history of recent administration of RTX and on his physical examination and laboratory findings, the patient was presumptively diagnosed as having acute serum sickness secondary to RTX and was treated with methylprednisolone of $1 \mathrm{mg} / \mathrm{kg} /$ day divided to 2 doses, ample analgesia with paracetamol, morphine, and fentanyl patches. He was immediately started on a wide-spectrum antibiotic at the beginning in view of suspected sepsis and was discontinued shortly after admission. His symptoms resolved within 48 hours after initiation of the above-mentioned treatment, his follow-up laboratory test results were normal. Four days later, he was discharged home and referred to a dermatology and allergy clinic where the treating physician has discussed with the primary physician the treatment options and possible complications for resuming the patient on RTX.

\section{Discussion}

Rituximab is a novel therapeutic agent for severe and recalcitrant pemphigus vulgaris (PV). ${ }^{19}$ In patients with PV, human antichimeric antibodies (e.g., RTX) are known to cause treatment failure and adverse effects especially with intravenous administration ${ }^{20}$.

However, there are not yet randomized trials assessing which protocol is better in terms of efficacy and safety. On the other hand, high dose regimens should be preferred instead of low-dose regimens, due to longer disease response. ${ }^{9}$

Rituximab-induced serum sickness (RISS) has been reported earlier in various autoimmune disorders including rheumatoid arthritis, Sjogren's syndrome, and hematological malignancies ${ }^{21}$. Typically, it has been explained by the presence of the murine component in RTX and B-cell lysis by forming complexes with antibodies due to the delivery of intracellular antigens to the serum which then precipitates systematically in the synovial membranes of joints ${ }^{22,23}$.

A 2015 literature review identified 33 reported cases associated with RTX where most of the reported cases were linked with an underlying rheumatologic condition $(\mathrm{n}=17,51.5 \%)$, most commonly Sjogren's syndrome (44.4\%). The classic triad of serum sickness (fever, rash, and arthralgia) was reported in 16 (48.5\%) cases. Time from drug exposure to symptom onset was significantly greater with the first doses of RTX compared 
to the second dose (mean time 10.00 vs. $4.05 \mathrm{~d}, \mathrm{P}=0.002$ ), and time to resolution was significantly greater for rheumatologic vs. hematological indications (mean time 2.50 vs. $1.00 \mathrm{~d}, \mathrm{P}=0.035$ ). ${ }^{24}$

A recent study has described the epidemiological and clinical characteristics of 37 cases of RISS reported in France. Serum sickness occurred mainly 12 days after the first injection (54\%). The most frequent manifestations were rheumatologic symptoms (92\%), fever (87\%), and skin lesions (78\%). The incidence was significantly higher when RTX was used for autoimmune diseases than for hematological malignancies. ${ }^{25}$

The role of RTX in severe refractory PV has been studied in the past few years. Some of the documented adverse effects include severe infections such as pneumonia, progressive multifocal leukoencephalopathy, anaphylaxis, Stevens-Johnson syndrome. ${ }^{19}$

In our case, the treating physician did not recommend resuming RTX to prevent any further severe reaction. Mainly, the diagnosis of serum sickness depends on clinical features. Other causes such as malignancy and any infection that can trigger serum sickness should be ruled out. In this case, investigations such as blood investigations (e.g., CBC, complement C3, C4, blood culture. etc.), ultrasonography abdomen, and urine analysis helped in ruling out malignancy and infectious potential causes. In our patient, clinical presentation, medication history, and quick response to treatment helped in making the diagnosis of RISS. Overall, all the clinical features, laboratory findings, quick response to corticosteroids were suggestive of serum sickness due to RTX (which was started two weeks ago) after excluding other possible causes.

\section{Conclusion}

Rituximab is considered an important treatment option in patients with moderate to severe refractory pemphigus vulgaris. RTX has been shown to be effective and well-tolerated in several autoimmune conditions. However, there are still concerns about its long-term adverse effects. Clinicians should be vigilant for potential acute or delayed serum sickness as possible adverse reactions to weigh the risks and benefits of continuing/reintroducing RTX for patients with pemphigus vulgaris. Further studies reporting the adverse effects of RTX are required to establish its safety and tolerability.

\section{Abbreviations}

CMV: Cytomegalovirus

COVID-19: Coronavirus disease

IBD: Inflammatory bowel disease

AIDS: Acquired immunodeficiency syndrome

ICU: Intensive care unit

IL-6: Interleukin 6

\section{Declarations}

\section{Ethics approval and consent to participate}

The article describes a case report. Therefore, no additional permission from our Ethics Committee was required.

\section{Consent for publication}

The consent for publication was obtained.

\section{Availability of data and material}

All data generated or analyzed during this study are included in this published article.

\section{Competing interests}

The authors declare that they have no competing interests. 


\section{Funding}

This study was not funded.

\section{Authors' contributions}

MYK, SMA, AJN: Data Collection, Literature Search, Manuscript Preparation

All authors read and approved the final manuscript

\section{Acknowledgments}

We thank the patient for allowing us to share his case. Open Access funding provided by the Qatar National Library

\section{References}

1. Waldmann TA. Monoclonal antibodies in diagnosis and therapy.Science . 1991;252(5013):1657-1662.

2. Cheson BD. Rituximab: clinical development and future directions. Expert opinion on biological therapy . 2002;2(1):97-110.

3. Onrust SV, Lamb HM, Balfour JAB. Rituximab. Drugs . 1999;58(1):79-88.

4. Weiner GJ. Rituximab: mechanism of action. Elsevier; 2010:115-123.

5. Fischer K, Cramer P, Busch R, et al. Bendamustine combined with rituximab in patients with relapsed and/or refractory chronic lymphocytic leukemia: a multicenter phase II trial of the German Chronic Lymphocytic Leukemia Study Group. Journal of clinical oncology . 2011;29(26):3559-3566.

6. Holle JU, Dubrau C, Herlyn K, et al. Rituximab for refractory granulomatosis with polyangiitis (Wegener's granulomatosis): comparison of efficacy in granulomatous versus vasculitic manifestations.Annals of the rheumatic diseases . 2012;71(3):327-333.

7. Roubaud-Baudron C, Pagnoux C, Meaux-Ruault N, et al. Rituximab maintenance therapy for granulomatosis with polyangiitis and microscopic polyangiitis. The Journal of rheumatology . 2012;39(1):125-130.

8. Knight C, Hind D, Brewer N, Abbott V. Rituximab (MabThera(r)) for aggressive non-Hodgkin's lymphoma: systematic review and economic evaluation. NIHR Health Technology Assessment programme: Executive Summaries. NIHR Journals Library; 2004.

9. Wang H-H, Liu C-W, Li Y-C, Huang Y-C. Efficacy of rituximab for pemphigus: a systematic review and meta-analysis of different regimens. Acta dermato-venereologica . 2015;95(8):928-932.

10. Maneiro R, Salgado E, Carmona L, Gomez-Reino JJ. Rheumatoid factor as predictor of response to abatacept, rituximab and tocilizumab in rheumatoid arthritis: systematic review and meta-analysis. Elsevier; 2013:9-17.

11. Ahmad A, Aggarwal A, Sharma D, et al. Rituximab for treatment of refractory/relapsing thrombotic thrombocytopenic purpura (TTP).American journal of hematology . 2004;77(2):171-176.

12. Dimopoulos MA, Anagnostopoulos A, Kyrtsonis M-C, et al. Primary treatment of Waldenstrom macroglobulinemia with dexamethasone, rituximab, and cyclophosphamide. Journal of Clinical Oncology . 2007;25(22):3344-3349.

13. Gupta N, Kavuru S, Patel D, et al. Rituximab-based chemotherapy for steroid-refractory autoimmune hemolytic anemia of chronic lymphocytic leukemia. Leukemia . 2002;16(10):2092.

14. Maloney DG, Grillo-Lopez AJ, White CA, et al. IDEC-C2B8 (Rituximab) anti-CD20 monoclonal antibody therapy in patients with relapsed low-grade non-Hodgkin's lymphoma. Blood . 1997;90(6):21882195 . 
15. Uzun S, Bilgic Temel A, Akman Karakaş A, et al. Efficacy and safety of rituximab therapy in patients with pemphigus vulgaris: first report from Turkey. International journal of dermatology . 2016;55(12):13621368.

16. Kimby E. Tolerability and safety of rituximab (MabThera@).Cancer treatment reviews . 2005;31(6):456473.

17. Maeda R, Kawasaki Y, Ohara S, Suyama K, Hosoya M. Serum sickness with refractory nephrotic syndrome following treatment with rituximab. CEN case reports . 2018;7(1):69-72.

18. Chung CH. Managing premedications and the risk for reactions to infusional monoclonal antibody therapy. The oncologist . 2008;13(6):725-732.

19. Awal G, Kaur S, Kaur J, Sharma S. Rituximab-induced serum sickness in pemphigus vulgaris. Journal of the Egyptian Women's Dermatologic Society . 2018;15(1):54-56.

20. Lunardon L, Payne AS. Inhibitory human antichimeric antibodies to rituximab in a patient with pemphigus. Journal of Allergy and Clinical Immunology . 2012;130(3):800-803.

21. Matsui T, Hidaka M, Kawakita T, et al. Serum sickness induced by rituximab infusion; report of two cases with hematological malignancies.[Rinsho ketsueki] The Japanese journal of clinical hematology . 2009;50(4):304-308.

22. Finger E, Scheinberg M. Development of serum sickness-like symptoms after rituximab infusion in two patients with severe hypergammaglobulinemia. JCR: Journal of Clinical Rheumatology . 2007;13(2):94-95.

23. D'Arcy CA, Mannik M. Serum sickness secondary to treatment with the murine-human chimeric antibody IDEC-C2B8 (rituximab). Arthritis 8 Rheumatism: Official Journal of the American College of Rheumatology . 2001;44(7):1717-1718.

24. Karmacharya P, Poudel DR, Pathak R, et al. Rituximab-induced serum sickness: a systematic review. Elsevier; 2015:334-340.

25. Bayer G, Agier M-S, Lioger B, et al. Rituximab-induced serum sickness is more frequent in autoimmune diseases as compared to hematological malignancies: A French nationwide study. European journal of internal medicine . 2019;67:59-64. 